\title{
Towards Decoding of Depersonalisation Disorder Using EEG: A Time Series Analysis Using CDTW
}

\author{
Abbas Salami \\ School of Computer Science and \\ Electronic Engineering \\ University of Essex \\ Colchester, UK \\ a.salami@essex.ac.uk
}

\author{
Javier Andreu-Perez \\ School of Computer Science and \\ Electronic Engineering \\ University of Essex \\ Colchester, UK \\ javier.andreu@essex.ac.uk
}

\author{
Helge Gillmeister \\ Department of Psychology \\ University of Essex \\ Colchester, UK \\ helge@essex.ac.uk
}

\begin{abstract}
Depersonalisation/derealisation refers to a transient psychological condition characterised by losing the sense of body ownership and feeling detached from the outside world. It is often accompanied by a lack of emotional responsiveness and sometimes memory fragmentation. Studies have shown the temporary occurrence of this condition among $34-70 \%$ of the general population during their life span. However, if the symptoms become consistent, they can be intolerable and can profoundly affect the quality of life in such an extent that it would be considered as one type of the dissociative disorders, depersonalisation disorder (DPD). Currently, there is no laboratory method to diagnose DPD, and studies have expressed a period of seven to 12 years for the correct diagnosis of DPD. We recently aimed to investigate DPD and its symptoms based on inexpensive and convenient electroencephalogram (EEG) neuroimaging technique, using calculation of event-related-potentials (ERPs) over the somatosensory cortex. We showed that DPD symptoms could be as a result of impairment in early (implicit) stages of information processing in the brain. We also introduced $P 45$ as a potential electrophysiological biomarker to study DPD. In this paper, we first replicated our results and then used P45 as a feature to discriminate between individuals with high and low tendency to DPD symptoms. We used Continuous Dynamic Time Warping (CDTW) to address the possible time shift and distortion in the ERP signals and to reach better classification performance. We reached $85 \%$ accuracy (Kappa 0.7) using leave-one-subject-out cross-validation, which confirms the feasibility for discrimination between DPD patients and a control group using EEG signals.
\end{abstract}

Keywords-depersonalisation, electrophysiological marker, EEG, ERP, SEP, classification

\section{INTRODUCTION}

Depersonalisation/derealisation is a psychological condition in which a person feels disengaged from the self as well as the surrounding [1]. The condition often happens in response to acute anxiety or traumatic situations as a defensive mechanism of the brain to protect the body organism [2-5]. Although the contact with reality stays intact $[6,7]$, the person's perception becomes unreal as if they are living in a dream [7]. The transient form of depersonalisation/derealisation has been observed in more than $50 \%$ of college students [8] and $34-70 \%$ of the nonclinical $[9,10]$. However, in cases the symptoms are chronic, it is considered as a type of dissociative disorders (Depersonalisation disorder (DPD); Diagnostic and Statistical Manual of Mental Disorders, 5th Edition (DSM-5) [11]).
Dissociative disorders are a group of mental disorders that are characterised by a lack of continuity between memory, thoughts, identity, actions, and the outside world. DPD can profoundly affect the quality of life for the patients and intervene in their daily social life. Patients complain about their life being "colourless", and they often have a lack of emotional responsiveness [7]. The symptoms of DPD can be divided into five main categories as in TABLE

Many attempts have been made in the literature to discover the underlying neurological basis of DPD symptoms (for review see $[7,12,13])$. Functional neuroimaging techniques such as functional magnetic resonance imaging (fMRI) and positron emission tomographic (PET) have shown abnormal activities of the insula and amygdala as sensory information processing units in DPD patients [14-16]. The insular cortex is a region located deep in the cerebral cortex [17], which is believed to be responsible for the integration and the processing of visceral signals [18] as well as the signals from our environment $[19,20]$. For instance, one dominant theory to explain emotional numbing in DPD defines a threshold for the level of anxiety (or any unpleasant salient stimuli) in which the emotional processing units, including anterior insula and amygdala, stop translating emotions into perceived feelings, and DPD is associated with abnormalities in triggering that threshold [3,21]. Nevertheless, there is no laboratory method to diagnose dissociative disorders at the moment, and due to the considerable circulation of DPD (1$2 \%[12,22,23])$, it is believed to be one of the most prevalent yet under-diagnosed psychological disorders [10, 24]. Since there are also overlaps between DPD and other psychological

TABLE I. FIVE MAJOR SYMPTOMS OF DPD (ADAPTED FROM $[6,26]$ AND OTHER ASSOCIATED PROCESSING DIFFERENCES)

\begin{tabular}{|l|l|}
\hline Symptom & Description \\
\hline $\begin{array}{l}\text { Disembodiment feelings } \\
\text { (desomatisation) }\end{array}$ & Lack of body ownership or loss of agency \\
\hline $\begin{array}{l}\text { Emotional numbing } \\
\text { (de-affectualisation) }\end{array}$ & Attenuation in emotional responsiveness \\
\hline $\begin{array}{l}\text { Anomalous subjective } \\
\text { recall } \\
\text { (de-ideation) }\end{array}$ & $\begin{array}{l}\text { Disassociation between an incident and } \\
\text { personal feeling in memory retrieval }\end{array}$ \\
\hline $\begin{array}{l}\text { Alienation from } \\
\text { surroundings } \\
\text { (derealisation) }\end{array}$ & $\begin{array}{l}\text { Detachment of the self from its } \\
\text { surroundings }\end{array}$ \\
\hline $\begin{array}{l}\text { Other symptoms and } \\
\text { processing differences }\end{array}$ & $\begin{array}{l}\text { Impaired attentional functioning and } \\
\text { processing speed or perceptual organisation }\end{array}$ \\
\hline
\end{tabular}


disorders such as depression and post-traumatic stress disorder (PTSD) [25], it currently takes seven to 12 years for DPD to be correctly diagnosed [10].

From several functional neuroimaging techniques, electroencephalogram (EEG) is of great importance due to its inexpensive and convenient nature. EEG represents electrical activities of a population of neurons in the human brain, which can be measured by electrodes situated on the surface of the scalp (Fig. 3 (B)). As well as its potential to classify several mental tasks [26-28], EEG has also shown promising capability in helping with the diagnosis of several mental disorders and psychological conditions [29]. Adler et al. [30] recently aimed to investigate DPD using EEG signals and tried to find potential electrophysiological biomarkers associated with DPD symptoms. In this paper, we first will replicate our previous results and will introduce the potential neurophysiological biomarker, which can help us to gain a better insight into the neural mechanism of DPD. Then we will use that biomarker as a potential feature to perform a classification task, which can help with the diagnosis of the disorder.

The rest of the paper is organised as follows. In section , we will introduce the participants and the experimental paradigm, and then we will explain our EEG signal preprocessing stages. In this section, we will also illustrate the replication of our previous findings correspond to the neural patterns of DPD, and we will show the use of our findings to perform a classification task between DPD patients and control subjects. The results will be presented in section and the future potentialities of our approach will be discussed in section

\section{EXPERIMENT AND METHODS}

\section{A. Participants}

The dataset used in this study was collected by [30]. The dataset includes 14 subjects with DPD and 15 sex and agematched control individuals. The evaluation of DPD symptoms was conducted based on the well-known Cambridge Depersonalisation Scale (CDS) [31], which is the most common and primary tool to quantify depersonalisation. CDS is a questionnaire that consists of 29 items such as "Parts of my body feel as if they didn't belong to me" or "My surroundings feel detached or unreal, as if there were a veil between me and the outside world". Participants are asked to rate each item in a 5-point scale based on the frequency of the symptoms over the last six months. The severity of DPD is then evaluated based on the overall score. Since this is a preliminary study in the evaluation of EEG signals as a diagnostic tool for DPD, the subjects with poor signal to noise ratio or close CDS score to the threshold were excluded from our analysis. The dataset used in this study contains 10 subjects with high CDS score and 10 subjects with low CDS score, which are referred to as DPD patients and control individuals, respectively.

\section{B. Experimental paradigm}

Fig. 1 shows the animated schematics of the two distinct categories of trials. Each trial started with $700 \mathrm{~ms}$ pre-stimuli in which subjects saw a picture of their own faces and a pencil. Then for $200 \mathrm{~ms}$, they received a tactile stimulation while they saw a picture of themselves being touched or not being touched (half of the trials for each condition) by the pencil. In no-touch trials, the pencil was next to the cheek, so the

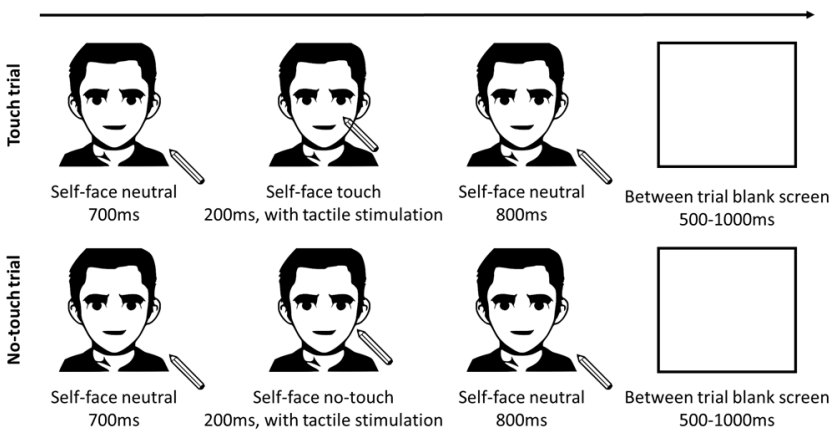

Fig. 1. Two types of trial design in the experiment

perceived distance that the pencil travelled would be the same across trials. Each trial ended with $800 \mathrm{~ms}$ post-stimuli in which subjects again saw their own faces and the pencil. The tactile stimulation was delivered to either left or right cheek (half of the trials for each) to cancel the possible effects of anatomical congruency between the viewed and felt touch. The EEG signals were recorded using 64-channels cap at $500 \mathrm{~Hz}$ sampling frequency.

\section{EEG preprocessing stages}

The continuous multi-channel EEG signals were filtered using a high-pass filter with $0.1 \mathrm{~Hz}$ cut-off frequency. The reason was to remove the DC offset and any low-frequency artifacts. EEG artifacts refer to any signals recorded during EEG acquisition with sources besides the brain, such as signals generated by blink, heart functioning, or temporary electrode displacement. Then we applied Cleanline method [32] to remove the $50 \mathrm{~Hz}$ line noise and its harmonics followed by a $40 \mathrm{~Hz}$ low-pass filter. In the frequency domain, the Cleanline method applies a regression model of a sinusoid signal with the frequency of the line noise on a time window of the original signal in the same frequency. Then by keeping the procedure over a sliding window, the method reconstructs the temporal representation of the line noise signal and subtracts it from the original signal. After that, bad EEG channels were identified manually by observing their temporal and frequency pattern. Electrodes containing excessive noise, constant pattern, or poor scalp-surface contact are considered as bad channels. They were removed and then interpolated using other existing channels. The whole multi-channel EEG was then re-referenced to the average of all electrodes to calculate the absolute voltage in each channel. This is because the voltages recorded by electrodes are differential voltages based on a reference (except ground). Finally, epochs containing blink or drift (linear trends) were visually detected and excluded from the dataset, and $100 \mathrm{~ms}$ pre-stimulus baseline was subtracted from the signal in each epoch.

\section{DPD brain responses and feature extraction}

Event-related potential (ERP) is a neural pattern generated by the brain in response to a specific event or stimulus [33]. In cases that the stimulus is tactile, it is referred to as somatosensory evoked potential (SEP). Each ERP signal contains several components that correspond to several brain mechanisms. Temporal average over several trials can be used to extract and visualise the components of ERP. The idea is based on the assumption that each ERP signal contains an additive white Gaussian noise (AGWN). So averaging over many trials can reject noise from the signal (because AGWN has zero mean) and therefore increase the signal to noise ratio. 


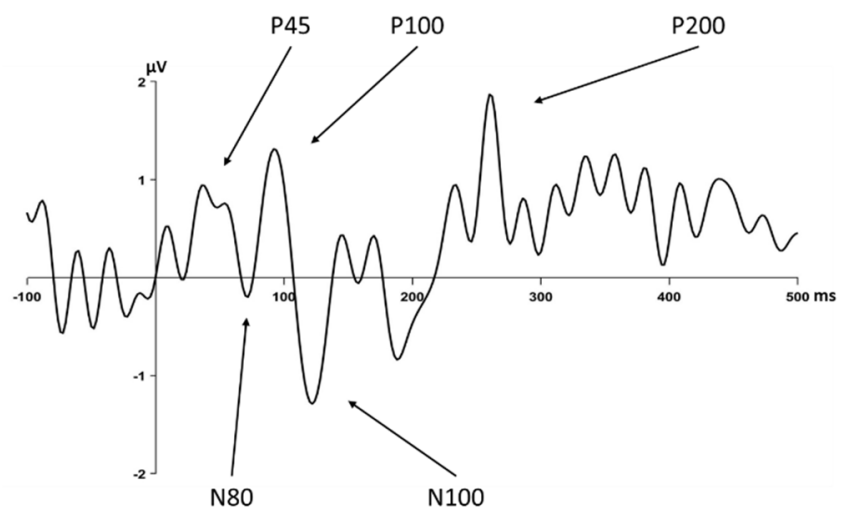

Fig. 2. Different SEP components

P45 is an early component of SEP, which appears as a positive peak around $45 \mathrm{~ms}$ after the stimulus onset and represents the autonomic (implicit) information processing [34]. Adler et al. [30] showed that there is a difference in the activation of $\mathrm{P} 45$ component in DPD patients in comparison with the control group. In this paper, we replicated the result of [30] in Fig. 4, which shows the average SEPs over touch (thick line) and no-touch trials (thin line) for DPD patients and the control group. Since the sources of P45 are located in centro-parietal regions of the brain (somatosensory cortex), electrodes placed on those areas were used to calculate the average SEPs (depicted in grey in Fig. 3 (B)). Due to the lateralization of brain functions, we expected to observe SEP components in response to left and right side tactile stimulation in the opposite hemispheres. So for stimulation to the left cheek (right cheek), we calculated SEP on the average of selected electrodes in the right (left) hemisphere. Then we obtained the average SEP response over both hemispheres. As

A)

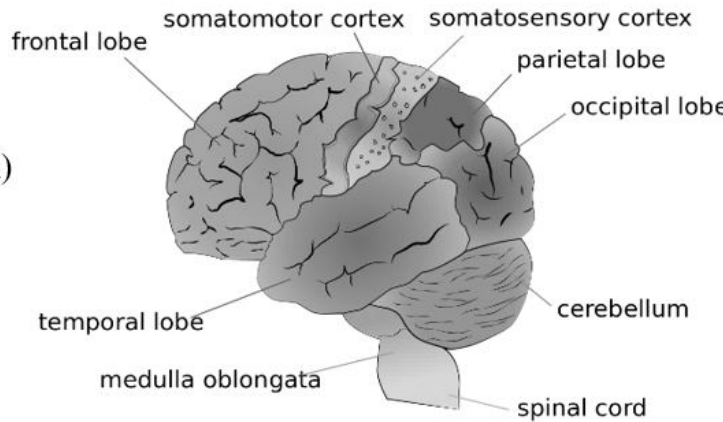

B)

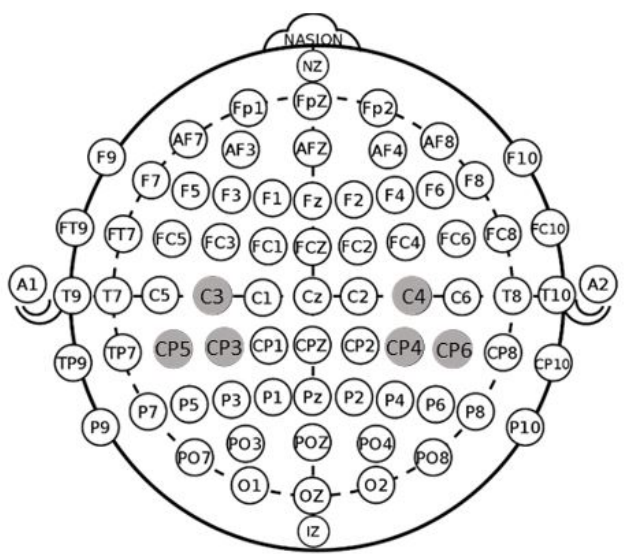

Fig. 3. Different brain regions (A) and the schematic of 64-channels cap (B) - filled in grey channels are the ones used to calculated SEPs

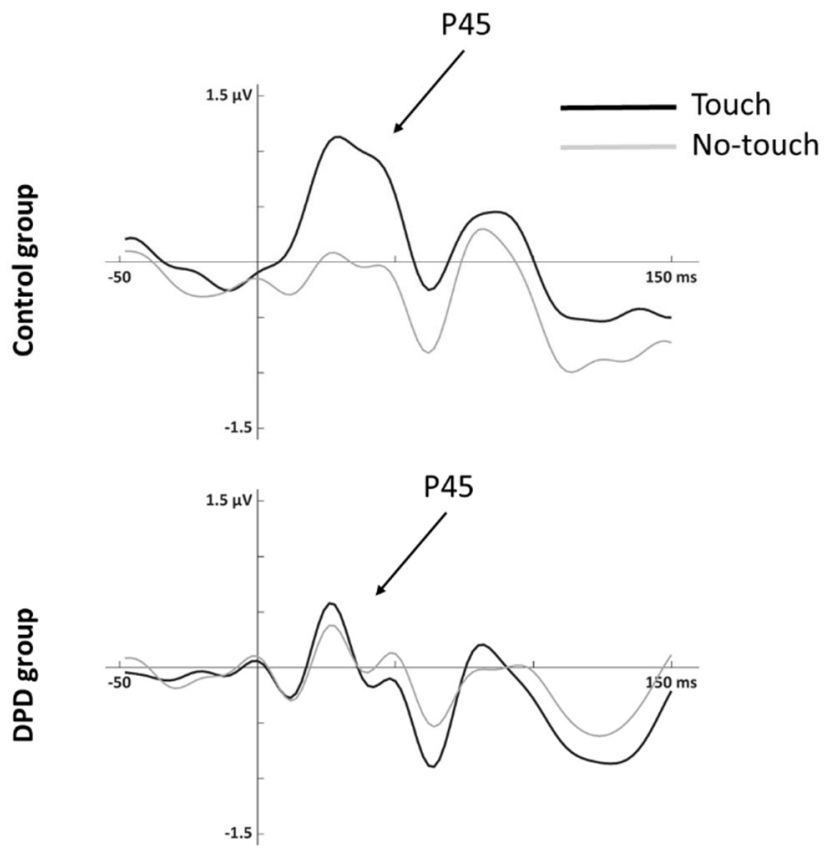

Fig. 4. Average SEPs for touch and no-touch trials in a cluster of electrodes located in the somatosensory cortex (electrodes marked in Fig. 3 (B))

can be seen in Fig. 4, there is a difference in the activation of P45 components in DPD patients compared with the control group.

The lack of $\mathrm{P} 45$ activation during touch trials represents impairment in early information processing in DPD patients $[35,36]$. Since there is a link between P45 and the sense of body ownership $[30,37,38]$, the observed lack of $P 45$ can be associated with the feeling of disembodiment in the DPD group [30].

\section{E. Extracted Features and classification method}

We used the difference found in the activation of P45 component of SEPs to perform classification task between DPD patients and the control group. We observed a difference between the average SEPs over touch and no-touch trials in the control group and not DPD patients. So we calculated the difference signal between touch and no-touch trials for the two groups in which it is a positive signal at around $45 \mathrm{~ms}$ poststimulus for the control group, while a fluctuated signal around zero for the DPD group.

In order to evaluate the potential of our founded electrophysiological biomarker to perform the classification task, we used leave-one-subject-out cross-validation. In each iteration, one subject was left for testing and the others for training. We calculated the average difference SRPs (touch vs no-touch) for all the DPD patients and all the control subjects in the training sets separately, and also for the test subject. So in each iteration of the leave-one-subject-out cross-validation, we ended up with 3 different time series for DPD group, control group and the test subject. Because the exact timing of the P45 component is not often clearly apparent, we considered a time window between 20 and $50 \mathrm{~ms}$ after the stimulus to analyse P45 component. To further address the possible distortion and time shift in the ERP components including $\mathrm{P} 45$, we then used continuous dynamic time warping (CDTW) [39, 40]. DTW is used for finding the 
similarity between to time series with possible delay and different speeds with respect to each other [41].

Consider two different multi-dimensional time series $A(t), t=1, \ldots, T_{1}$ and $B(t), t=1, \ldots, T_{2}$. The general idea is to find a warping map $\psi=\left[\psi_{1}(t), \psi_{2}(t)\right]^{T}$ between the two time series which minimizes the following similarity measure:

$$
\begin{aligned}
& D(A, B)= \\
& \sum_{t=2}^{T} d\left(\left(A\left(\psi_{1}(t-1)\right), B\left(\psi_{2}(t-1)\right)\right),\left(A\left(\psi_{1}(t)\right), B\left(\psi_{2}(t)\right)\right)\right) \\
& =\sum_{t=2}^{T}\left\|\overrightarrow{A\left(\psi_{1}(t)\right) B\left(\psi_{2}(t)\right)}-\overrightarrow{A\left(\psi_{1}(t-1)\right) B\left(\psi_{2}(t-1)\right)}\right\|^{2}
\end{aligned}
$$

where $\|$.$\| is the Frobenius norm. In the warping or matching$ map $\psi$, a point $A\left(\psi_{1}(t)\right)$ corresponds to a point $B\left(\psi_{2}(t)\right)$ for $t=1, \ldots, T$. Minimization of the similarity measure $D$ can be done using dynamic programming in the so-called a multistage decision process [42] based on the following recursive formula:

$$
\begin{aligned}
& D(t)=\min _{\psi_{1}(t-1)}\{D(t-1)+ \\
& \left.d\left(\left(A\left(\psi_{1}(t-1)\right), B\left(\psi_{2}(t-1)\right)\right),\left(A\left(\psi_{1}(t)\right), B\left(\psi_{2}(t)\right)\right)\right)\right\}
\end{aligned}
$$

Note that in general $A(t)$ and $B(t)$ can be samples of two continues time series, however, in case that the two time series are discrete, $\psi_{1}(t)$ and $\psi_{2}(t)$ can only take values on $\left\{1, \ldots, T_{1}\right\}$ and $\left\{1, \ldots, T_{2}\right\}$ respectively. Therefore, the warping map can be derived from the distance matrix calculated as follows:

$$
\begin{aligned}
& D(i, j)=\|A(i)-B(j)\| \\
& +\min \{D(i-1, j), D(i, j-1), D(i-1, j-1) \\
& i=1, \ldots, T_{1} \\
& j=1, \ldots, T_{2}
\end{aligned}
$$

Then a warping path (alignment path) is found, starting from the top right corner of the distance matrix to the bottom left corner, by selecting the cells with the minimum value in each step from the adjacent cells and keeping the slope of the path always non-negative. The similarity measure between the two time series is then the sum of distances on the warping path. An example of the warping plane and the warping path is illustrated in Fig. 5. In this example, the aligned signals after DTW are as follows:

$$
\begin{aligned}
& A^{\prime}=\left(A^{1}, A^{1}, A^{1}, A^{1}, A^{2}, A^{2}, A^{2}, A^{3}, A^{4}, A^{5}, A^{6}, A^{7}, A^{7}, A^{8}, A^{9}, A^{10}\right) \\
& B^{\prime}=\left(B^{1}, B^{2}, B^{3}, B^{4}, B^{4}, B^{5}, B^{6}, B^{6}, B^{7}, B^{7}, B^{7}, B^{7}, B^{8}, B^{9}, B^{9}, B^{10}\right)
\end{aligned}
$$

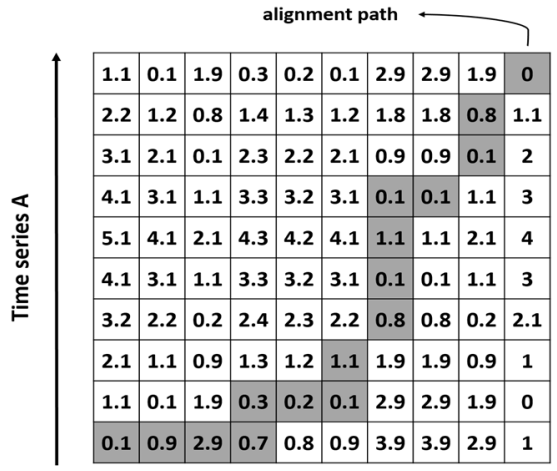

Time series B

Fig. 5. Sample illustration of the distance matrix and the alignment path in DTW
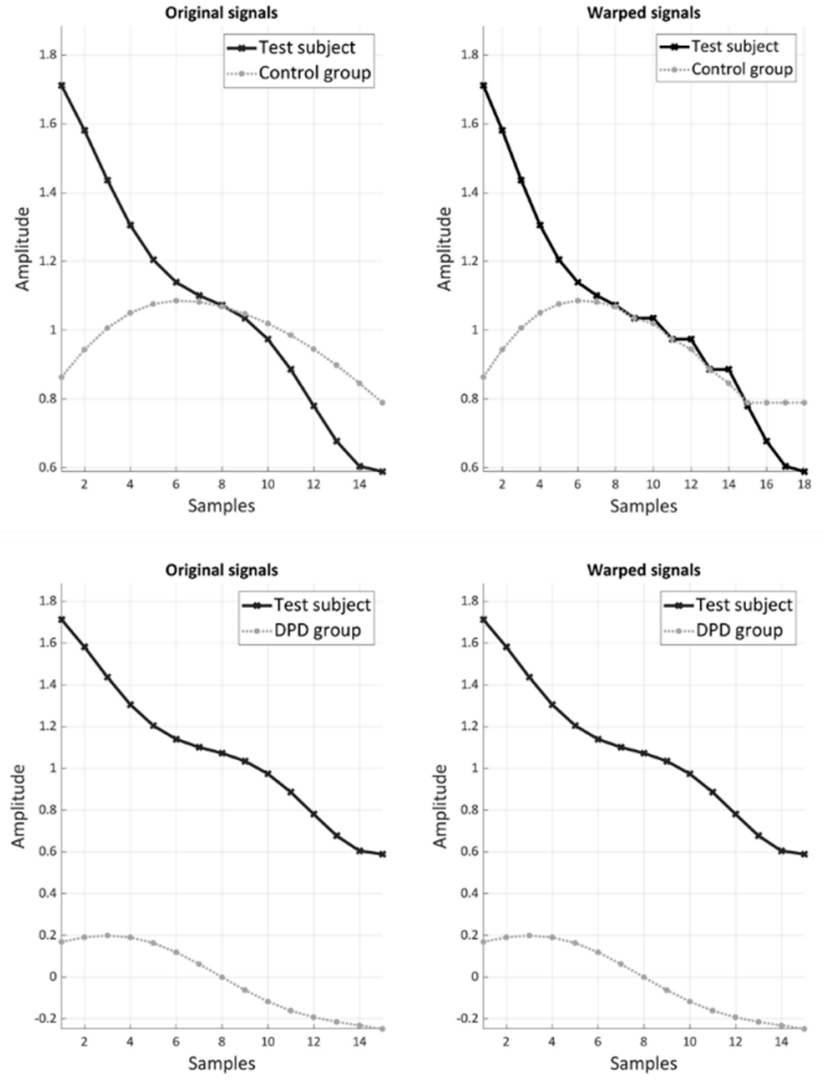

Fig. 6. Signal alignment for a test subject (C19) in comparison with the average SEP of the control group and the DPD group

As mentioned earlier, $A(t)$ and $B(t)$ can be a subsample of two continues time series. CDTW is a continuous version of DTW in which each point of one time series can be matched with any value between two samples of the other time series (more details can be found in [39]).

Fig. 6 shows an example of the extracted signal for control subjects, DPD patients, and the test subject. It also shows the aligned signals after applying CDTW. For this example, the similarity measure between the signal of the test subject and the control group and DPD group is 12.4742 and 18.9643,

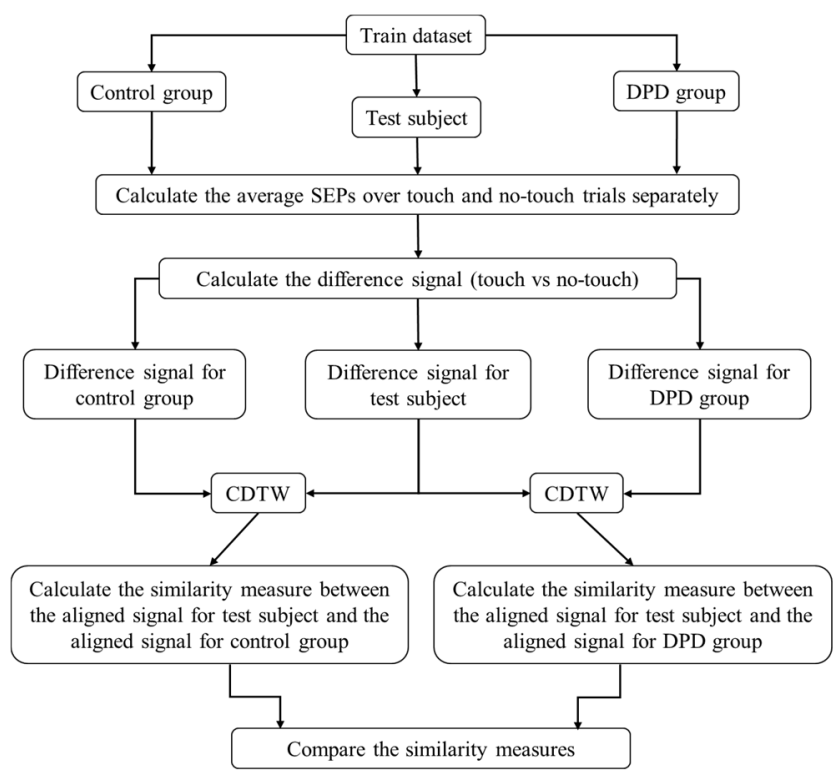

Fig. 7. Flowchart of the classification procedure 
TABLE II. PARTICIPANTS CDS SCORE AND THE CLASSIFICATION RESULTS USING PROPOSED ELECTROPHYSIOLOGICAL BIOMARKER (P45)

\begin{tabular}{|c|c|c|c|c|c|}
\hline ID & $\begin{array}{c}\text { True } \\
\text { class }\end{array}$ & $\begin{array}{c}\text { CDS } \\
\text { score }\end{array}$ & $\begin{array}{c}\text { Similarity } \\
\text { measure with } \\
\text { the control } \\
\text { group }\end{array}$ & $\begin{array}{c}\text { Similarity } \\
\text { measure with } \\
\text { the DPD } \\
\text { group }\end{array}$ & Result \\
\hline C10 & Ctrl. & 10 & 3.9061 & 3.9663 & Ctrl. \\
\hline C15 & Ctrl. & 9 & 1.4856 & 18.0678 & Ctrl. \\
\hline C17 & Ctrl. & 18 & 4.1491 & 29.6794 & Ctrl. \\
\hline C19 & Ctrl. & 19 & 12.4742 & 18.9643 & Ctrl. \\
\hline C2 & Ctrl. & 19 & 0.1501 & 9.7086 & Ctrl. \\
\hline C20 & Ctrl. & 12 & 0.1976 & 9.8148 & Ctrl. \\
\hline C4 & Ctrl. & 4 & 4.2445 & 4.9838 & Ctrl. \\
\hline C66 & Ctrl. & 19 & 7.6045 & 28.8936 & Ctrl. \\
\hline C70 & Ctrl. & 11 & 19.0275 & 48.8561 & Ctrl. \\
\hline C71 & Ctrl. & 17 & 10.1552 & 35.2177 & Ctrl. \\
\hline D11 & DPD & 71 & 26.3729 & 0.7552 & DPD \\
\hline D14 & DPD & 199 & 11.6940 & 5.3986 & DPD \\
\hline D15 & DPD & 101 & 19.7699 & 1.0860 & DPD \\
\hline D18 & DPD & 72 & 83.6372 & 34.2489 & DPD \\
\hline D3 & DPD & 91 & 23.1570 & 1.1153 & DPD \\
\hline D5 & DPD & 99 & 8.6665 & 9.4076 & Ctrl. \\
\hline D6 & DPD & 140 & 67.6014 & 24.0648 & DPD \\
\hline D9 & DPD & 97 & 4.4002 & 2.3384 & DPD \\
\hline D13 & DPD & 111 & 2.6640 & 10.8761 & Ctrl. \\
\hline D69 & DPD & 98 & 3.0716 & 26.8930 & Ctrl. \\
\hline Number of subjects correctly classified & & 17 & \\
\hline & Total number of subjects & & 20 & \\
\hline & Accuracy \% (Kappa value) & $\mathbf{8 5 \% ~ ( 0 . 7 ) ~}$ \\
\hline
\end{tabular}

respectively. Therefore, the test subject was assigned to the control group.

\section{RESULTS}

TABLE shows the classification results using leaveone-subject-out cross-validation. TABLE also contains the similarity measures between the signals for each subject and the two classes. We were able to reach $85 \%$ classification accuracy for our dataset. We claimed that there are a distortion and time shift in the signals. So in order to show the effectiveness of using CDTW in our scenario, we also excluded CDTW and calculated the number of correctly classified subjects based on the Euclidean distances (Euclidean distances can be calculated since all the time series have same number of samples) as a similarity measure on original SEP signals without alignment. In addition, one other possible approach would be to consider the peak value in the chosen time window as the P45 component. If the value for the signal peak in the time window was closer to the signal peak of the control group, it would be assigned to the control group, otherwise the DPD group. The comparison of our proposed classification procedure with the above mentioned approaches is presented in TABLE

\section{Conclusions}

In this paper, we aimed to investigate the neurological correlates of DPD based on EEG and find an electrophysiological biomarker to serve as a potential feature
TABLE III. COMPARISON OF OUR CLASSIFICATION APPROACH WITH OTHER POSSIBLE APPROACHES

\begin{tabular}{|c|c|c|}
\hline $\begin{array}{c}\text { Classification } \\
\text { strategy }\end{array}$ & $\begin{array}{c}\text { Number of correctly } \\
\text { classified subjects }\end{array}$ & $\begin{array}{c}\text { Classification } \\
\text { accuracy (\%) }\end{array}$ \\
\hline $\begin{array}{c}\text { Using peak amplitude } \\
\text { Without signal } \\
\text { alignment }\end{array}$ & 15 & 75 \\
\hline $\begin{array}{c}\text { Proposed approach } \\
\text { Propo }\end{array}$ & 16 & $\mathbf{8 5}$ \\
\hline
\end{tabular}

to discriminate between individuals with high and low level of depersonalisation symptoms. This task is of great importance since DPD has a prevalence of around $1-2 \%$ in the general population, which is comparable to that of schizophrenia and obsessive-compulsive disorder (OCD). We demonstrated the lack of P45 activation as one of the components of SEP in DPD patients. P45 has shown to be responsible for implicit sensory information processing. Besides, the role of this early component in the sense of body ownership has been confirmed. In this regard, we recently argued that the feeling of disembodiment or loss of sense of bodily self in DPD patients could be as a result of impairment in early implicit stages of information processing. In this paper, we replicated our previous results and used P45 component of SEP as a feature to perform the classification task between DPD patients and a control group. We used CDTW to tackle the problem of possible time shift and distortion in signals around P45. We were able to reach $85 \%$ accuracy (Kappa value of 0.7 ), which shows the effectiveness of our findings regarding P45 to be a valuable electrophysiological biomarker to help with the diagnosis of DPD.

In this study, we used ERP and its components which are derived from temporal analysis of EEG signals. This is while some studies have confirmed abnormal activities in the theta band $(4-8 \mathrm{~Hz})$ of the EEG power spectrum in DPD patients [6, 43]. Although their results might be related to Adler et al. findings since the low frequencies of the EEG power spectrum are believed to play the central role in the formation of early ERP components [44], but further studies on EEG power spectrum in DPD patients is needed. Besides, since transient depersonalisation is a common phenomenon during the life span, developing a system to track depersonalisation state and its severity could be of great importance to help with the prevention of the chronic type. Analysis of the EEG signals of the DPD patients with more sophisticated signal processing and machine learning algorithms as well as addressing a solution for tracking depersonalisation state are our future directions for investigation around this psychological disorder.

\section{REFERENCES}

[1] M. L. Phillips and M. Sierra, "Depersonalization disorder: a functional neuroanatomical perspective," Stress, vol. 6, no. 3, pp. 157-165, 2003.

[2] E. Shilony and F. K. Grossman, "Depersonalization as a defense mechanism in survivors of trauma," J. Trauma. Stress, vol. 6, no. 1, pp. 119-128, 1993.

M. Sierra and G. E. Berrios, "Depersonalization: neurobiological perspectives," Biol. Psychiatry, vol. 44, no. 9, pp. 898-908, 1998. E. Hunter, M. L. Phillips, T. Chalder, M. Sierra, and A. David, "Depersonalisation disorder: a cognitive-behavioural 
conceptualisation," Behav. Res. Ther., vol. 41, no. 12, pp. 14511467, 2003.

[5] D. J. Stein and D. Simeon, "Cognitive-affective neuroscience of depersonalization," CNS spectrums, vol. 14, no. 9, pp. 467-471, 2009.

[6] E. Hollander, J. L. Carrasco, L. S. Mullen, S. Trungold, C. M. DeCaria, and J. Towey, "Left hemispheric activation in depersonalization disorder: a case report," Biol. Psychiatry, vol. 31, no. 11, pp. 1157-1162, 1992 .

[7] M. Sierra and A. S. David, "Depersonalization: a selective impairment of self-awareness," Conscious. Cogn., vol. 20, no. 1, pp. 99-108, 2011.

[8] J. Dixon, "Depersonalization phenomena in a sample population of college students," The British journal of psychiatry, vol. 109, no. 460 , pp. $371-375,1963$.

[9] Y. Aderibigbe, R. Bloch, and W. Walker, "Prevalence of depersonalization and derealization experiences in a rural population," Soc. Psychiatry Psychiatr. Epidemiol., vol. 36, no. 2, pp. 63-69, 2001.

[10] E. C. Hunter, J. Charlton, and A. S. David, "Depersonalisation and derealisation: assessment and management," $B M J$, vol. 356, p. j745, 2017.

[11] American Psychiatric Association, Diagnostic and statistical manual of mental disorders (BMC Med). 2013, pp. 133-137.

[12] E. C. Hunter, M. Sierra, and A. S. David, "The epidemiology of depersonalisation and derealisation," Soc. Psychiatry Psychiatr. Epidemiol., vol. 39, no. 1, pp. 9-18, 2004.

[13] A. Salami, J. Andreu-Perez, and H. Gillmeister, "Symptoms of depersonalisation/derealisation disorder as measured by brain electrical activity: A systematic review," Neurosci. Biobehav. Rev., vol. 118, pp. 524-537, 2020, doi: 10.1016/j.neubiorev.2020.08.011.

[14] M. L. Phillips et al., "Depersonalization disorder: thinking without feeling," Psychiatry Research: Neuroimaging, vol. 108, no. 3, pp. 145-160, 2001.

[15] J. W. Hopper, P. A. Frewen, B. A. Van der Kolk, and R. A. Lanius, "Neural correlates of reexperiencing, avoidance, and dissociation in PTSD: Symptom dimensions and emotion dysregulation in responses to script - driven trauma imagery," $J$. Trauma. Stress, vol. 20, no. 5, pp. 713-725, 2007.

[16] N. Medford, M. Sierra, A. Stringaris, V. Giampietro, M. J. Brammer, and A. S. David, "Emotional experience and awareness of self: functional MRI studies of depersonalization disorder," Front. Psychol., vol. 7, p. 432, 2016.

[17] L. Q. Uddin, J. S. Nomi, B. Hebert-Seropian, J. Ghaziri, and O. Boucher, "Structure and function of the human insula," Journal of clinical neurophysiology: official publication of the American Electroencephalographic Society, vol. 34, no. 4, p. 300, 2017.

[18] A. D. Craig, "How do you feel--now? The anterior insula and human awareness," Nature reviews neuroscience, vol. 10, no. 1, 2009 .

[19] K. O. Bushara, J. Grafman, and M. Hallett, "Neural correlates of auditory-visual stimulus onset asynchrony detection," $J$. Neurosci., vol. 21, no. 1, pp. 300-304, 2001.

[20] K. O. Bushara, T. Hanakawa, I. Immisch, K. Toma, K. Kansaku, and M. Hallett, "Neural correlates of cross-modal binding," Nat. Neurosci., vol. 6, no. 2, p. 190, 2003.

[21] E.-L. Jay, M. Sierra, F. Van den Eynde, J. C. Rothwell, and A. S. David, "Testing a neurobiological model of depersonalization disorder using repetitive transcranial magnetic stimulation," Brain stimulation, vol. 7, no. 2, pp. 252-259, 2014.

[22] M. Michal, M. E. Beutel, J. Jordan, M. Zimmermann, S. Wolters, and T. Heidenreich, "Depersonalization, mindfulness, and childhood trauma," The Journal of nervous and mental disease, vol. 195 , no. 8, pp. 693-696, 2007.

[23] W. E. Lee, C. H. Kwok, E. C. Hunter, M. Richards, and A. S. David, "Prevalence and childhood antecedents of depersonalization syndrome in a UK birth cohort," Soc. Psychiatry Psychiatr. Epidemiol., vol. 47, no. 2, pp. 253-261, 2012.

[24] M. Michal, M. E. Beutel, and T. G. Grobe, "How often is the Depersonalization-Derealization Disorder (ICD-10: F48. 1) diagnosed in the outpatient health-care service?," Zeitschrift fur
Psychosomatische Medizin und Psychotherapie, vol. 56, no. 1, pp. 74-83, 2010.

[25] C. Armour, A. A. Contractor, P. A. Palmieri, and J. D. Elhai, "Assessing latent level associations between PTSD and dissociative factors: Is depersonalization and derealization related to PTSD factors more so than alternative dissociative factors?," Psychol. Inj. Law, vol. 7, no. 2, pp. 131-142, 2014.

[26] J. Andreu-Perez, F. Cao, H. Hagras, and G.-Z. Yang, "A selfadaptive online brain-machine interface of a humanoid robot through a general type-2 fuzzy inference system," IEEE Transactions on Fuzzy Systems, vol. 26, no. 1, pp. 101-116, 2016.

[27] A. Gupta et al., "On the Utility of Power Spectral Techniques With Feature Selection Techniques for Effective Mental Task Classification in Noninvasive BCI," IEEE Transactions on Systems, Man, and Cybernetics: Systems, 2019.

[28] A. Gupta et al., "A hierarchical meta-model for multi-class mental task based brain-computer interfaces," Neurocomputing, vol. 389 , pp. 207-217, 2020

[29] W. J. Bosl, "The Emerging Role of Neurodiagnostic Informatics in Integrated Neurological and Mental Health Care," The Neurodiagnostic Journal, vol. 58, no. 3, pp. 143-153, 2018.

[30] J. Adler, N. Schabinger, M. Michal, M. E. Beutel, and H. Gillmeister, "Is that me in the mirror? Depersonalisation modulates tactile mirroring mechanisms," Neuropsychologia, vol. 85 , pp. $148-158,2016$

[31] M. Sierra and G. E. Berrios, "The Cambridge Depersonalisation Scale: A new instrument for the measurement of depersonalisation," Psychiatry Res., vol. 93, no. 2, pp. 153-164, 2000 .

[32] T. Mullen. "NITRC: CleanLine: Tool/Resource http://www.nitrc.org/projects/cleanline (accessed March 22, 2015).

[33] S. J. Luck, An introduction to the event-related potential technique. MIT press, 2014.

[34] R. C. Josiassen, C. Shagass, R. A. Roemer, S. Slepner, and B. Czartorysky, "Early cognitive components of somatosensory event-related potentials," Int. J. Psychophysiol., vol. 9, no. 2, pp. 139-149, 1990.

[35] C. W. Quaedflieg et al., "Early emotional processing deficits in depersonalization: An exploration with event-related potentials in an undergraduate sample," Psychiatry Research: Neuroimaging, vol. 212, no. 3, pp. 223-229, 2013.

[36] N. Schabinger, H. Gillmeister, S. Berti, M. Michal, M. E. Beutel, and J. Adler, "Detached and distracted: ERP correlates of altered attentional function in depersonalisation," Biol. Psychol., vol. 134, pp. 64-71, Apr 2018, doi: 10.1016/j.biopsycho.2018.02.014.

[37] N. Otsuru et al., "Sensory incongruence leading to hand disownership modulates somatosensory cortical processing," Cortex, vol. 58, pp. 1-8, 2014.

[38] S. Rigato, A. J. Bremner, H. Gillmeister, and M. J. Banissy, "Interpersonal representations of touch in somatosensory cortex are modulated by perspective," Biol. Psychol., p. 107719, 2019.

[39] M. E. Munich and P. Perona, "Continuous dynamic time warping for translation-invariant curve alignment with applications to signature verification," in Proceedings of the Seventh IEEE International Conference on Computer Vision, 1999, vol. 1: IEEE, pp. 108-115.

[40] P. Micó. "Continuous Dynamic Time Warping." https://www.mathworks.com/matlabcentral/fileexchange/16350continuous-dynamic-time-warping (accessed January 27, 2020).

[41] H. Sakoe and S. Chiba, "Dynamic programming algorithm optimization for spoken word recognition," IEEE Trans. Acoust., vol. 26 , no. 1 , pp. 43-49, 1978.

[42] R. Bellman, "Dynamic programming princeton university press princeton," New Jersey Google Scholar, 1957.

[43] E. B. Raimo, R. A. Roemer, M. Moster, and Y. Shan, "Alcoholinduced depersonalization," Biol. Psychiatry, vol. 45, no. 11, pp. 1523-1526, 1999.

[44] A. P. Burgess, "Towards a unified understanding of event-related changes in the EEG: the firefly model of synchronization through cross-frequency phase modulation," PLoS One, vol. 7, no. 9, 2012 . 